\title{
Comparison of the Substrate Preferences of $\omega 3$ Fatty Acid Desaturases for Long Chain Polyunsaturated Fatty Acids
}

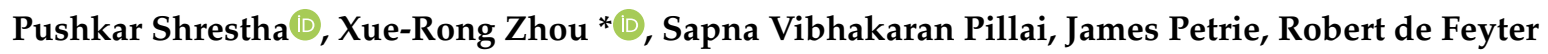 \\ and Surinder Singh \\ CSIRO Agriculture \& Food, Canberra, ACT 2601, Australia; Pushkar.Shrestha@csiro.au (P.S.); \\ Sapna.Vibhakaranpillai@csiro.au (S.V.P.); James.Petrie@csiro.au (J.P.); Robert.Defeyter@csiro.au (R.d.F.); \\ Surinder.Singh@csiro.au (S.S.) \\ * Correspondence: Xue-Rong.Zhou@csiro.au; Tel.: +61-2-6246-5455
}

Received: 24 May 2019; Accepted: 21 June 2019; Published: 22 June 2019

\begin{abstract}
Omega-3 long chain polyunsaturated fatty acids ( $\omega 3$ LC-PUFAs) such as eicosapentaenoic acid (EPA; 20:5 $\omega 3$ ) and docosahexaenoic acid (DHA; 22:6w3) are important fatty acids for human health. These $\omega 3$ LC-PUFAs are produced from their $\omega 3$ precursors by a set of desaturases and elongases involved in the biosynthesis pathway and are also converted from $\omega 6$ LC-PUFA by omega-3 desaturases ( $\omega 3 \mathrm{D}$ s). Here, we have investigated eight $\omega 3$-desaturases obtained from a cyanobacterium, plants, fungi and a lower animal species for their activities and compared their specificities for various C18, C20 and C22 $\omega 6$ PUFA substrates by transiently expressing them in Nicotiana benthamiana leaves. Our results showed hitherto unreported activity of many of the $\omega 3 \mathrm{Ds}$ on $\omega 6$ LC-PUFA substrates leading to their conversion to $\omega 3$ LC-PUFAs. This discovery could be important in the engineering of EPA and DHA in heterologous hosts.
\end{abstract}

Keywords: Omega-3 desaturase; long-chain polyunsaturated fatty acids; substrate specificity; EPA; DHA

\section{Introduction}

Long chain polyunsaturated fatty acids such as arachidonic acid (ARA; 20:4w6), eicosapentaenoic

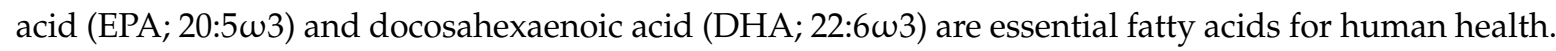
Arachidonic acid is mainly located in the brain, skeletal muscles and liver, while EPA and DHA are rich in the brain, retina and skin. LC-PUFAs are divided into $\omega 6$ and $\omega 3$ LC-PUFAs, depending upon the positioning of the last double bond in the fatty acid chain, either at the sixth carbon from the terminal methyl end in $\omega 6$ fatty acids or at the third carbon in $\omega 3$ fatty acids. Beneficial effects on infant growth and development have been shown for w6 LC-PUFAs such as ARA, but they have also been associated with blood coagulating (pro-thrombotic) and pain initiating (pro-inflammatory) properties, whereas $\omega 3$ LC-PUFAs have anti-thrombotic and anti-inflammatory properties. Thus, higher $\omega 6 / \omega 3$ fatty acid ratios are related to several health problems such as obesity [1], diabetes, inflammatory-autoimmune diseases, mood disorders [2] and depression. On the other hand, the occurrence of low-level cardiovascular diseases in Inuit and Japanese populations has been attributed to the higher level of $\omega 3 / \omega 6$ ratios in their bodies, which is believed to be due to the consumption of $\omega 3$ fatty acid-rich sea foods in their daily diet [3]. Recently, Simopoulus [1] has reported higher $\omega 6 / \omega 3$ ratios in populations of the Western world. The ratio is alarmingly high in urban India due to the consumption mainly of land-sourced foods which are generally rich in w6-PUFA and where there is little access to $\omega 3$ LC-PUFA rich marine foods. Clear health benefits have been shown for 
w3 LC-PUFAs such as EPA and DHA for cardiovascular diseases, hyperlipidaemia, hypertension, inflammatory diseases, and their potential benefits for diabetes, mood disorders, cancer and Alzheimer disease have also been reported.

The human body produces only a low level of LC-PUFA from the consumption of shorter chained fatty acids from plant and animal sources. Because most land-based foods are richer in shorter chain $\mathrm{C} 18 \omega 6$ fatty acids than $\mathrm{C} 18 \omega 3$ fatty acids, their consumption leads to the production of a higher proportion of $\omega 6$ LC-PUFAs and a lower proportion of the healthier $\omega 3$ LC-PUFAs in the human body. Therefore, the Food and Agriculture Organization of the United Nations, the World Health Organization and the European Food Safety Authority have recommended an intake of a minimum of $250 \mathrm{mg}$ of EPA + DHA daily to maintain good health [4]. Currently, marine fish and marine fish oil are the main sources of $\omega 3$ LC-PUFAs. However, fish do not synthesize LC-PUFA in their body but acquire these fatty acids by consuming w3-PUFA-rich microalgae and phytoplankton. Certain marine flora have a set of special desaturases and elongases which convert short chain fatty acids to $\omega 3$ LC-PUFAs. In addition, some fatty acid desaturases have the ability to introduce a double bond at the $\omega 3$ position of different $\omega 6$ fatty acids and convert the $\omega 6$ PUFAs into $\omega 3$ PUFAs. These desaturases are therefore known as $\omega 3$-desaturases ( $\omega 3 \mathrm{Ds})$.

The demand for $\omega 3$ LC-PUFAs is increasing for nutraceutical, pharmaceutical and aquaculture feeding purposes, while the marine fish stock, which is the main source, is declining globally. As an economical and sustainable alternative source of these fatty acids in oil seeds, fish oil-like levels of EPA [5] and DHA [6] were developed in the oil seed crop, camelina by the expression of a set of microalgal genes. There are some $\omega 6$ LC-PUFA intermediates in EPA camelina, while the levels of $\omega 6$ LC-PUFA are very low in DHA-camelina [6] and DHA-Arabidospsis [7] seeds, although they are rich in $\omega 6$ PUFA (18:2 $\omega 6)$ substrate. The role of the seed $\omega 3$ Ds in converting the native and the engineered $\omega 6$ substrates into $\omega 3$ LC-PUFAs is not clear, and it is important to understand this mechanism. Nevertheless, $\omega 3$ Ds have also shown important roles in the responses to different environmental stresses, such as temperature, drought, light, salinity, wounding and diseases [8].

Several $\omega 3$ Ds have been identified in plants and microorganisms and they have shown diverse phylogeny, functional characteristics and substrate preferences. For example, $\omega 3$ Ds obtained from Fusarium spp. [9] and Mortierella alpina [10] and expressed in yeast catalysed the desaturation of C18 $\omega 6$ PUFAs and, at lower activities, C20 $\omega 6$ PUFA substrates. In contrast, the $\omega 3 \mathrm{D}$ from an EPA-rich fungus, Saprolgenia diclina, when expressed in yeast, did not recognize C18 substrates and was active only on C20 $\omega 6$ fatty acid substrates [11]. More broadly, the $\omega 3 \mathrm{D}$ from another EPA-rich fungus, Phytophthora infestans [12], had greater activity on C20 and C22 fatty acid substrates than on C18 substrates but was active on all of those tested [13]. The FAT-1 $\omega 3 \mathrm{D}$ of the EPA-containing animal species Caenorhabditis elegans [14] was highly active on C18 fatty acids as well as the C20 substrate, dihomo-gamma-linolenic acid (20:3w6) [15]. Somewhat differently, when expressed in yeast, Brassica napus $\omega 3$ D exhibited broad specificity for C16-C22 substrates [16] and the Aspergillus nidulans bifunctional oleoyl $\Delta 12 /$ linoleoyl $\omega 3$ desaturase converted $\mathrm{C} 18$ and $\mathrm{C} 20 \omega 6$ substrates to their respective $\omega 3$ fatty acids [17]. For others, the fungus Neurospora crassa produces ALA [18], possibly involving a $\omega 3 \mathrm{D}$, and Synechocystis sp. $\omega 3 \mathrm{D}$ produces $\alpha$-linolenic acid (18:3 $\omega 3$ ) and stearidonic acid (18:4 $\omega 3$ ) [19], but their $\omega 3$ D specificities have not been investigated. Other special activities of $\omega 3 \mathrm{D}$ had been also reported. For example, M. alpina $1 S-4 \omega 3 \mathrm{D}$ also inserted a double bond at $\Delta 15$-position of medium chain fatty acid 16:2 $2^{9,12}$, resulting in the formation of unusual 16:39,12,15 [20]. Chang et al. [21] proposed that thraustochytrid $\omega 3 \mathrm{D}$ could also work on odd-chain LC-PUFA 21:5 $\omega-5$ ) leading to unusual 21:6 $\omega 2$.

However, our understanding of $\omega 3 \mathrm{D}$ is still fragmentary [8], and the diversity of $\omega 3 \mathrm{D}$ substrate specificity from various organisms is unclear or even unknown. A few of these $\omega 3$ Ds have been tested in plant cells. Although the ability of $\omega 3$ Ds to desaturate C18 and C20 substrates has been studied in a few species, their specificities for C22 PUFA substrates are still not understood.

Here, we have used a transient expression system in N. benthamiana leaves to investigate a range of eight $\omega 3$ Ds obtained from a cyanobacterium, plants, fungi and the nematode $C$. elegans for 
their activities and compared their specificities for various C18, C20 and C22 LC-PUFA substrates. Our results showed an unexpected wide range of $\omega 3 \mathrm{D}$ activity on various $\omega 6$ LC-PUFA substrates leading to their conversion to $\omega 3$ LC-PUFAs. Some of these activities were not reported previously. We also observed that these $\omega 3$ Ds had different substrate preferences.

\section{Results}

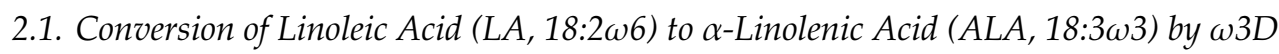

Eight individual $\omega 3$ Ds were transiently expressed in N. benthamiana leaves in the presence of the p19 viral gene silencing suppressor to reduce co-suppression and extend $\omega 3 \mathrm{D}$ production. The introduction of $\mathrm{p} 19$ alone was used as a control. N. benthamiana leaves possess endogenous $\omega 3 \mathrm{D}$ activity which converts endogenously present linoleic acid to linolenic acid (Figure 1), and therefore all test samples were compared to the control. We first investigated the activities of transiently expressed $\omega 3$ Ds by measuring the increase in the conversion rate of endogenous LA to ALA, compared to the p19-only infiltrated (control) tissues. The p19-only infiltrated tissues showed a $85.0 \pm 2.5 \%$ conversion of LA to ALA, while the addition of A. thaliana $\omega 3 \mathrm{D}$ (At- $\omega 3 \mathrm{D})$, B. napus $\omega 3 \mathrm{D}$ (Bn- $\omega 3 \mathrm{D})$, A. nidulans $\omega 3 \mathrm{D}$

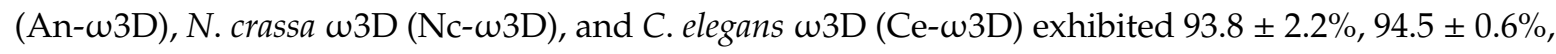
$93.2 \pm 0.8 \%, 93.1 \pm 1.4 \%$, and $91.8 \pm 2.8 \%$ conversions of LA, respectively, indicating the expression of the introduced $\omega 3 \mathrm{D}$ genes in the leaves and their activities for LA (Figure 1). Among all these, Bn- $\omega 3 \mathrm{D}$ had the highest conversion rate for LA, which was $9.5 \%$ higher than the endogenous $\omega 3 \mathrm{D}$ activity of the leaves (p19 only). However, the conversion rates with P. infestans $\omega 3 \mathrm{D}$ (Pi- $\omega 3 \mathrm{D} ; 84.1 \pm 2.0 \%$ ) and S. diclina $\omega 3 \mathrm{D}$ (Sd- $\omega 3 \mathrm{D} ; 83.8 \pm 2.2 \%$ ) were essentially the same as the control, indicating little or no activity for those enzymes on LA. Synechocystis $\omega 3 \mathrm{D}$ (Ss- $\omega 3 \mathrm{D} ; 87.9 \pm 3.3 \%$ ) showed a slightly increased conversion of LA.

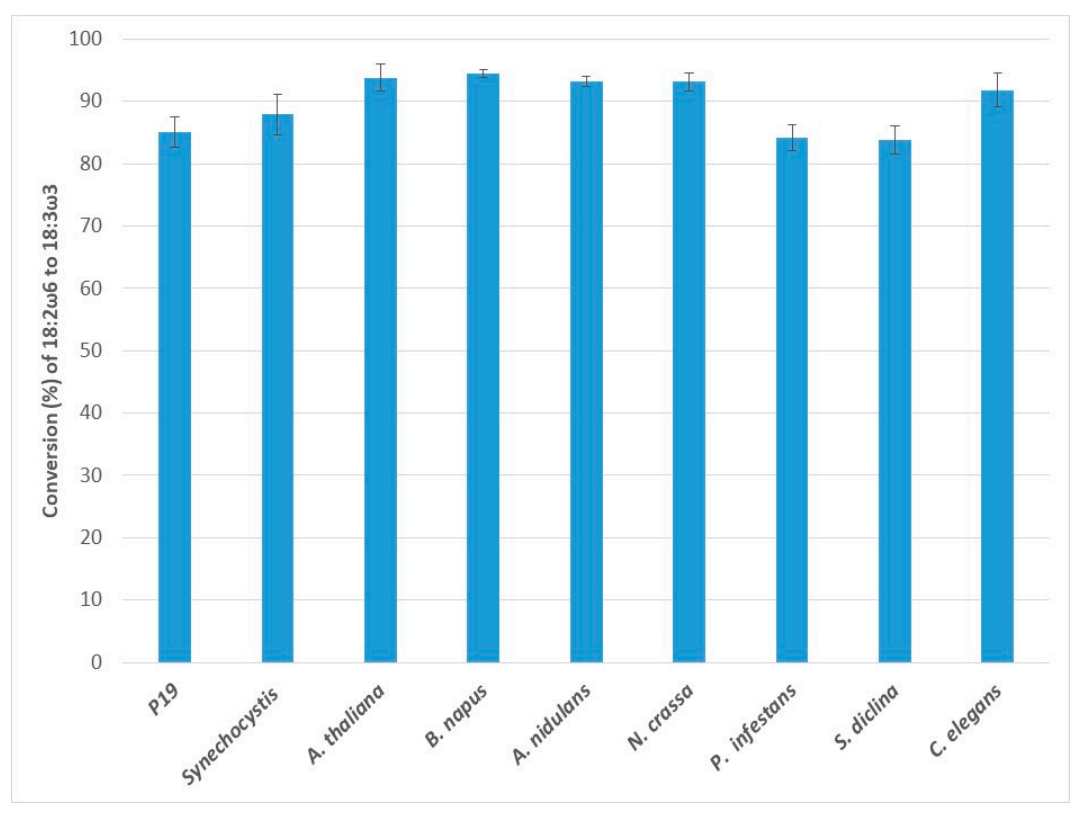

Figure 1. Enzymatic activities of transiently expressed $\omega 3$ Ds from various sources in N. benthamiana leaves on 18:2 $\omega 6$ substrate. $\omega 3 \mathrm{D}$ activities were determined by measuring the conversion rate of

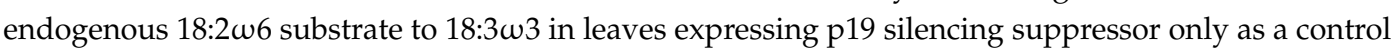
or the appropriate $\omega 3 \mathrm{D}$ with $\mathrm{p} 19$. The error bars denote the standard deviations of the means from triplicate assays. 


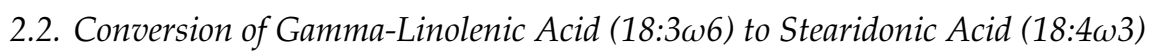

Application of gamma-linolenic acid (GLA) to the $\omega 3 \mathrm{D}$ expressing leaf tissues resulted in the production of stearidonic acid, indicating the $\omega 3 \mathrm{D}$ activities on another $\mathrm{C} 18 \omega 6$ substrate, GLA (Figure 2). The conversion rate of the endogenous $N$. benthamiana leaf $\omega 3 \mathrm{D}$ was $4.2 \pm 2.9 \%$. The transient expression of exogenous $\omega 3$ Ds clearly showed higher conversion rates for GLA than the endogenous $\omega 3 \mathrm{D}$ in the control and were $55.2 \pm 9.7 \%, 37.3 \pm 2.2 \%, 25.5 \pm 6.0 \%, 15.3 \pm 0.6 \%, 27.2 \pm 4.2 \%$ and $24.6 \pm 6.3 \%$ for Ce- $\omega 3 \mathrm{D}, \mathrm{An}-\omega 3 \mathrm{D}, \mathrm{Nc}-\omega 3 \mathrm{D}, \mathrm{Pi}-\omega 3 \mathrm{D}, \mathrm{At}-\omega 3 \mathrm{D}$ and Bn- $\omega 3 \mathrm{D}$, respectively. The conversion rate of Ce- $\omega 3 \mathrm{D}$ for GLA was the highest, being 13.1-fold higher than the endogenous conversion efficiency of the leaf $\omega 3 \mathrm{D}$. Sd- $\omega 3 \mathrm{D}$ and Ss- $\omega 3 \mathrm{D}$ showed no activity on GLA; i.e., no increase was observed in the conversion rate compared to the p19 control.

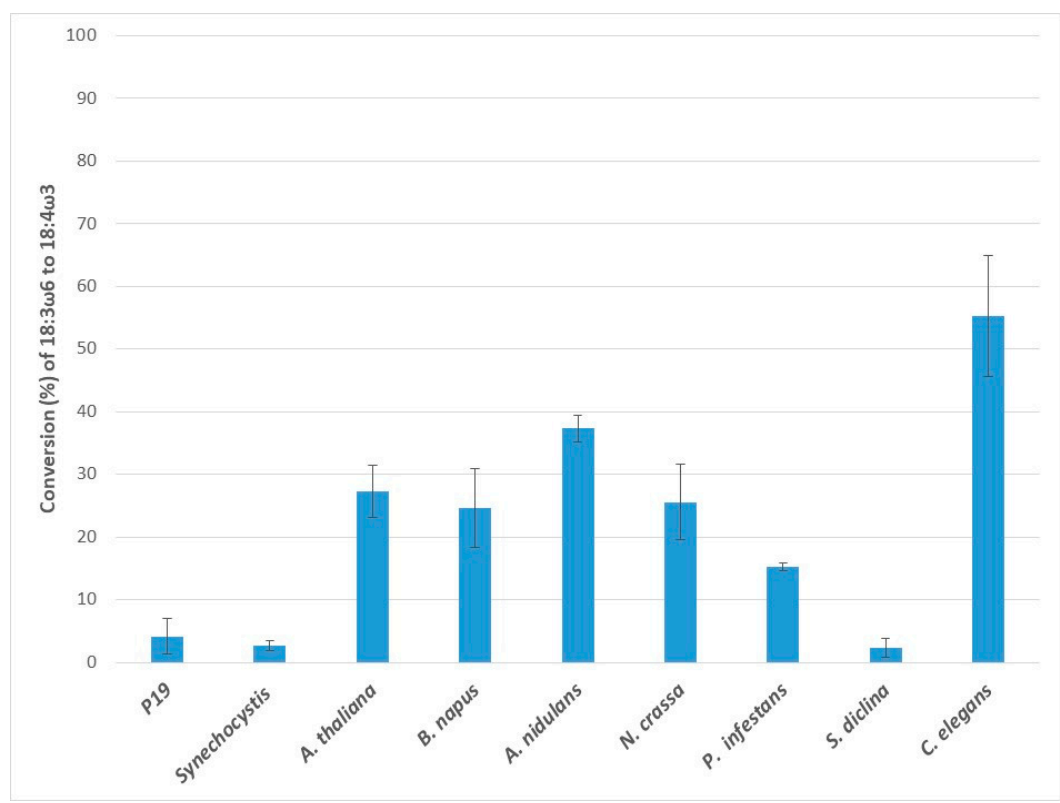

Figure 2. Enzymatic activities of transiently expressed $\omega 3$ Ds from various sources in N. benthamiana leaves on 18:3 $\omega 6$ substrate. $\omega 3 \mathrm{D}$ activities were determined by measuring the conversion rate of

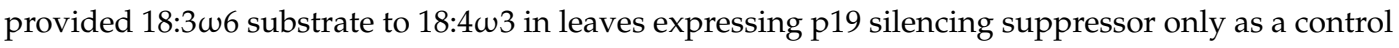
or the appropriate $\omega 3 \mathrm{D}$ with $\mathrm{p} 19$. The error bars denote the standard deviations of the means from triplicate assays.

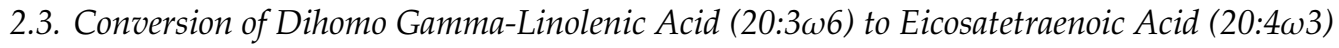

The application of dihomo gamma-linolenic acid (DGLA) to the $\omega 3$ D-expressing leaf tissues resulted in the production of eicosatetraenoic acid (ETA), indicating activities of $\omega 3 \mathrm{D}$ on the C20 $\omega 6$ substrate (Figure 3). The endogenous $\omega 3 \mathrm{D}$ had a low conversion rate for DGLA. Except for Ss- $\omega 3 \mathrm{D}$, the other seven $\omega 3$ Ds showed a high level of activity for DGLA compared to the endogenous activity of the leaves $(1.4 \pm 1.7 \%)$. Pi- $\omega 3 \mathrm{D}$ showed a very high conversion rate for DGLA to ETA $(71.7 \pm 3.2 \%)$, in contrast to its lower level of activity with GLA. Although GLA activity was absent with Sd- $\omega 3 \mathrm{D}$, it showed a strong preference for DGLA $(66.8 \pm 3.6 \%)$, and Ce- $\omega 3 \mathrm{D}(61.8 \pm 2.5 \%)$, Nc- $\omega 3 \mathrm{D}(28.1 \pm 7.7 \%)$ and An- $\omega 3 \mathrm{D}(39.2 \pm 3.0 \%)$ also showed high DGLA activities. Interestingly, the plant $\omega 3 \mathrm{Ds}$, Bn- $\omega 3 \mathrm{D}$ and At- $\omega 3 \mathrm{D}$ revealed conversion efficiencies of $45.5 \pm 6.0$ and $41.7 \pm 5.0 \%$, respectively, for the C20 substrate DGLA, which is not a native substrate in plants. Among the enzymes, Pi- $\omega 3 \mathrm{D}$ showed the highest conversion rate for DGLA. Again, just like its GLA response, Ss- $\omega 3 \mathrm{D}$ did not show any activity with DGLA. 


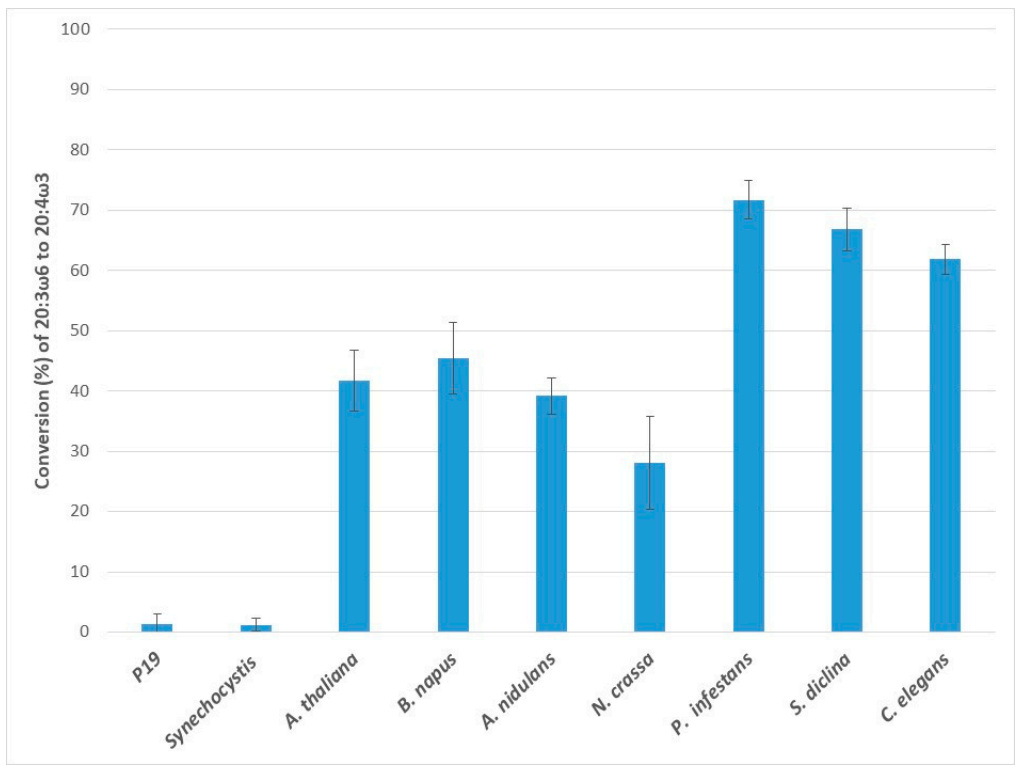

Figure 3. Enzymatic activities of transiently expressed $\omega 3 \mathrm{D}$ s from various sources in $N$. benthamiana leaves on 20:3 $\omega 6$ substrate. $\omega 3 \mathrm{D}$ activities were determined by measuring the conversion rate of

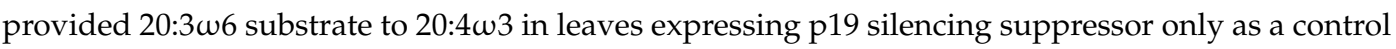
or the appropriate $\omega 3 \mathrm{D}$ with $\mathrm{p} 19$. The error bars denote the standard deviations of the means from triplicate assays.

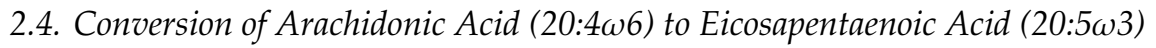

When arachidonic acid (ARA) was supplied, all eight $\omega 3$ Ds investigated were able to convert it to EPA (Figure 4). Again, the endogenous $\omega 3 \mathrm{D}$ in p19 control had a low conversion rate for ARA. The highest activity level was observed for Ce- $\omega 3 \mathrm{D}$ with the conversion rate of $69.7 \pm 3.0 \%$, followed by Pi- $\omega 3 \mathrm{D}(66.4 \pm 5.2 \%)$ and Sd- $\omega 3 \mathrm{D}(65.7 \pm 2.0 \%)$, while the plant $\omega 3 \mathrm{Ds}, \mathrm{Bn}-\omega 3 \mathrm{D}$ and At $-\omega 3 \mathrm{D}$ exhibited $47.0 \pm 10.5 \%$ and $39.0 \pm 0.9 \%$ conversion of ARA. Nc- $\omega 3 \mathrm{D}(11.6 \pm 3.7 \%)$ and An- $\omega 3 \mathrm{D}(17.2 \pm 2.7 \%)$ had lower levels of conversion with ARA. Ss- $\omega 3 \mathrm{D}$ had a $7.6 \pm 2.2 \%$ conversion rate for ARA, in contrast to an absence of activities with GLA and DGLA.

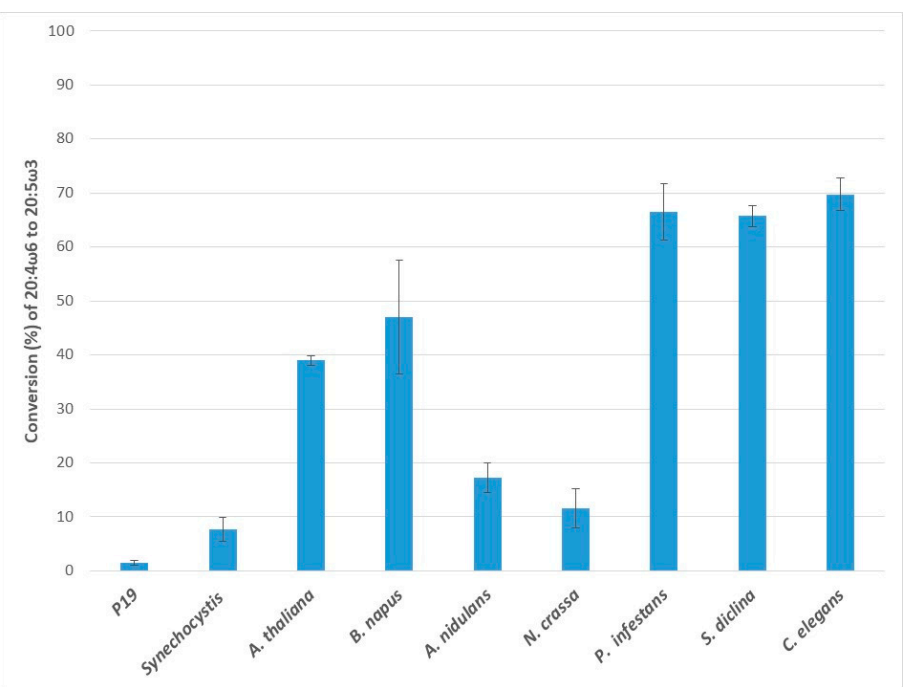

Figure 4. Enzymatic activities of transiently expressed $\omega 3 \mathrm{Ds}$ from various sources in $N$. benthamiana leaves on 20:4 $\omega 6$ substrate. $\omega 3 \mathrm{D}$ activities were determined by measuring the conversion rate of

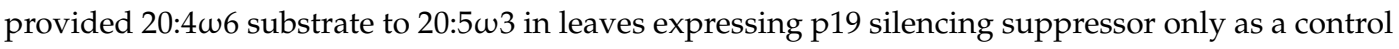
or the appropriate $\omega 3 \mathrm{D}$ with $\mathrm{p} 19$. The error bars denote the standard deviations of the means from triplicate assays. 


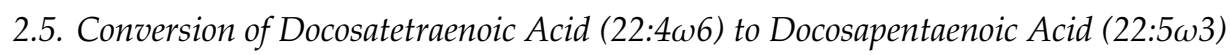

When the C22:4w6 fatty acid docosatetraenoic acid (DTA) was supplied, all the $\omega 3$ Ds tested were observed to desaturate DTA to the $\omega 3$ product docosapentaenoic acid (DPA) (Figure 5). The endogenous $\omega 3 \mathrm{D}$ in p19 control had little activity on the provided C22 substrate. High levels of conversion were observed for Pi- $\omega 3 \mathrm{D}(83.2 \pm 2.3 \%)$, Sd- $\omega 3 \mathrm{D}(79.5 \pm 0.7 \%)$, and Ce- $\omega 3 \mathrm{D}(78.0 \pm 1.5 \%)$, as well as the plant $\omega 3 \mathrm{Ds}, \mathrm{Bn}-\omega 3 \mathrm{D}(71.2 \pm 1.6 \%)$ and At- $\omega 3 \mathrm{D}(66.6 \pm 7.0 \%)$. Ss- $\omega 3 \mathrm{D}(10.2 \pm 0.3 \%), \mathrm{Nc}-\omega 3 \mathrm{D}(9.6 \pm 7.2 \%)$ and An-w3D $(9.3 \pm 2.0 \%)$ showed lower rates of DTA conversion.

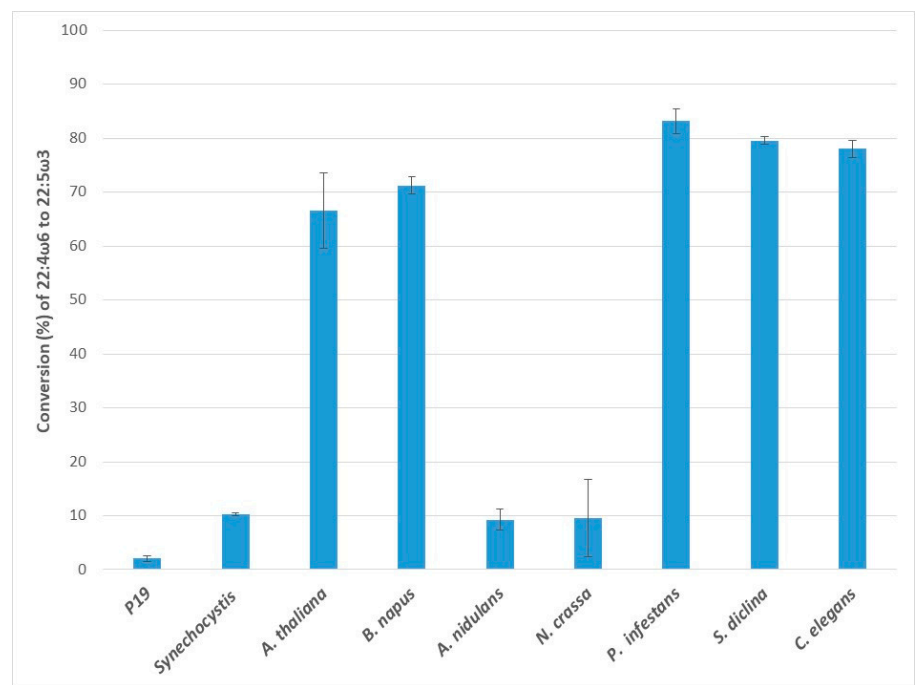

Figure 5. Enzymatic activities of transiently expressed $\omega 3 \mathrm{Ds}$ from various sources in N. benthamiana leaves on 22:4 $\omega 6$ substrate. $\omega 3 \mathrm{D}$ activities were determined by measuring the conversion rate of

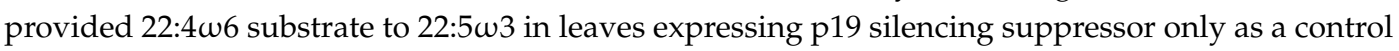
or the appropriate $\omega 3 \mathrm{D}$ with $\mathrm{p} 19$. The error bars denote the standard deviations of the means from triplicate assays.

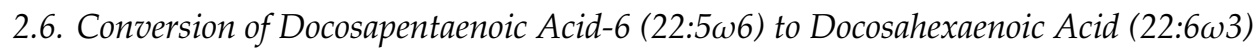

When the C22:5 $\omega 6$ fatty acid docosapentaenoic acid (DPAw6) was supplied, all the $\omega 3$ Ds studied here converted it to DHA (Figure 6). The endogenous $\omega 3 \mathrm{D}$ in p19 control had minor activity on the provided C22 substrate, as observed above. The highest rate of conversion was seen for Pi- $\omega 3 \mathrm{D}$ $(79.5 \pm 5.6 \%)$, followed by Sd- $\omega 3 \mathrm{D}(70.1 \pm 8.4 \%), \mathrm{Ce}-\omega 3 \mathrm{D}(61.9 \pm 5.5 \%)$, Bn- $\omega 3 \mathrm{D}(61.4 \pm 3.5 \%)$ and

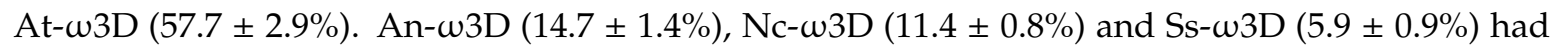
lower conversion rates for DPA $\omega 6$. 


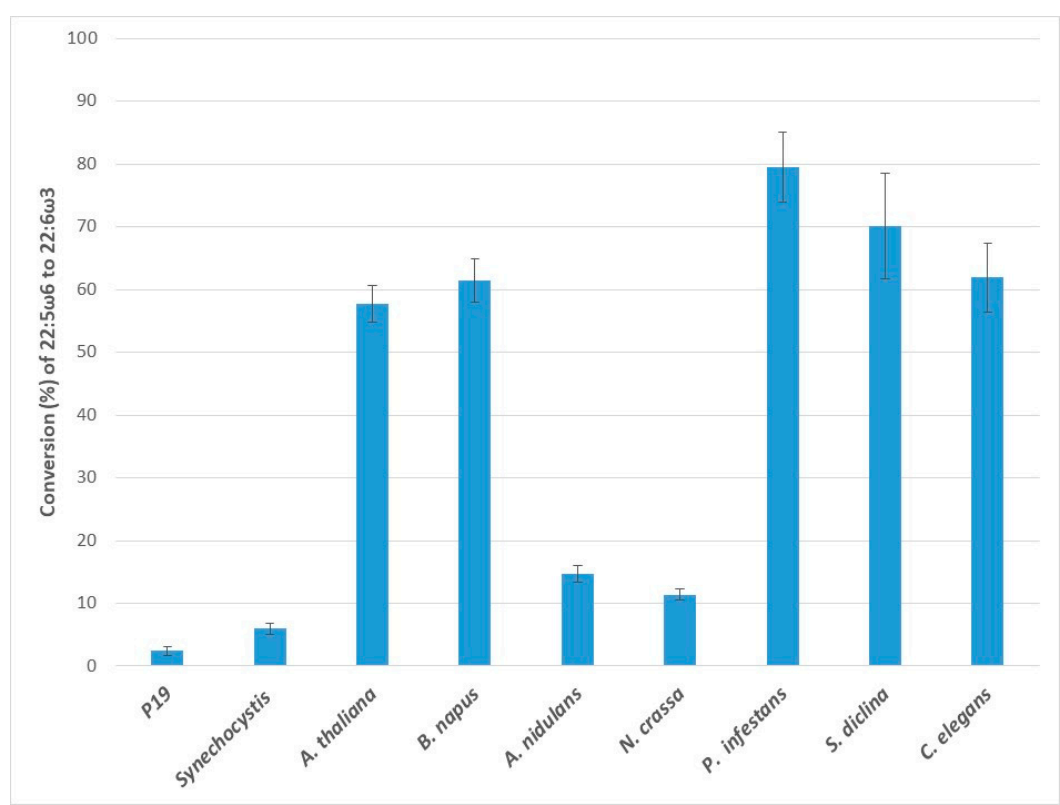

Figure 6. Enzymatic activities of transiently expressed $\omega 3 \mathrm{Ds}$ from various sources in $N$. benthamiana leaves on 22:5 $\omega 6$ substrate. $\omega 3 \mathrm{D}$ activities were determined by measuring the conversion rate of provided 22:5 $\omega 6$ substrate to 22:6w3 in leaves expressing p19 silencing suppressor only as a control or the appropriate $\omega 3 \mathrm{D}$ with $\mathrm{p} 19$. The error bars denote the standard deviations of the means from triplicate assays.

\subsection{Competition Among $\omega 6$ Fatty Acid Substrates for $\omega 3 D$ s}

In nature, multiple fatty acid substrates are available to a $\omega 3 \mathrm{D}$ at the same time. The activity for one substrate might be affected by the presence of other substrates, and the enzyme might have preferential selectivity for one substrate over others. Here, we attempted to measure the conversion efficiency of each substrate during competition among a pool of five different substrates (18:3w6, 20:3 $\omega 6,20: 4 \omega 6,22: 4 \omega 6,22: 5 \omega 6)$. Unlike the individual substrate assays, Ss- $\omega 3$ D showed the highest

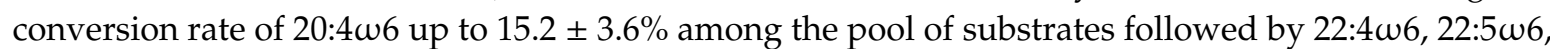

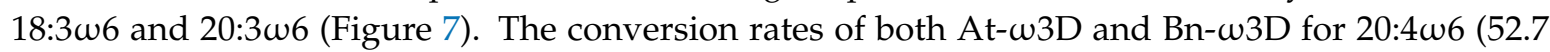
$\pm 5.0 \%, 67.7 \pm 4.2 \%)$ and $22: 4 \omega 6(54.0 \pm 4.8 \%, 65.4 \pm 3.7 \%)$ during substrate competition were the highest among all the substrates, followed by 22:5w6, 20:3w6 and 18:3w6). An- $\omega 3 \mathrm{D}$ and Nc- $\omega 3 \mathrm{D}$ demonstrated the preference for the $\mathrm{C} 18$ or $\mathrm{C} 20$ substrates. The An- $\omega 3 \mathrm{D}$ activities for 20:3 $\omega 6$ was the highest among these five substrates with the conversion rate of $49.4 \pm 6.0 \%$, followed by the activities

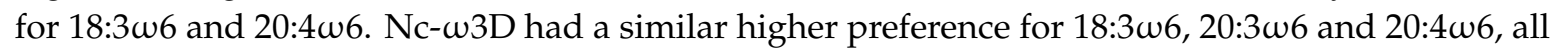

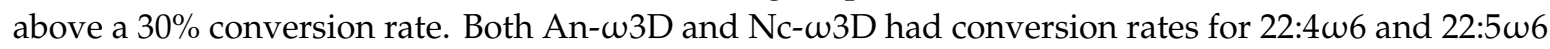
of only around $10 \%$. Pi- $w 3 \mathrm{D}$ had higher activities for both C20 and C22 substrates with a conversion rate above $70 \%$, but low activity for $18: 3 \omega 6$ with a conversion rate of $24.3 \pm 0.5 \%$. Sd- $\omega 3 \mathrm{D}$ also showed a higher preference for both C20 and C22 substrates, with a conversion rate at $60 \%$ or above, while a very low preference for 18:3w6, with a conversion rate of $3.8 \pm 2.3 \%$. Interestingly, Ce- $\omega 3 \mathrm{D}$ showed strong activities for all the tested C18, C20 and C22 substrates, ranging from 58\% to $75 \%$. Among these eight $\omega 3 \mathrm{Ds}, \mathrm{Ce}-\omega 3 \mathrm{D}$ had the highest conversion rate for 18:3w6. 


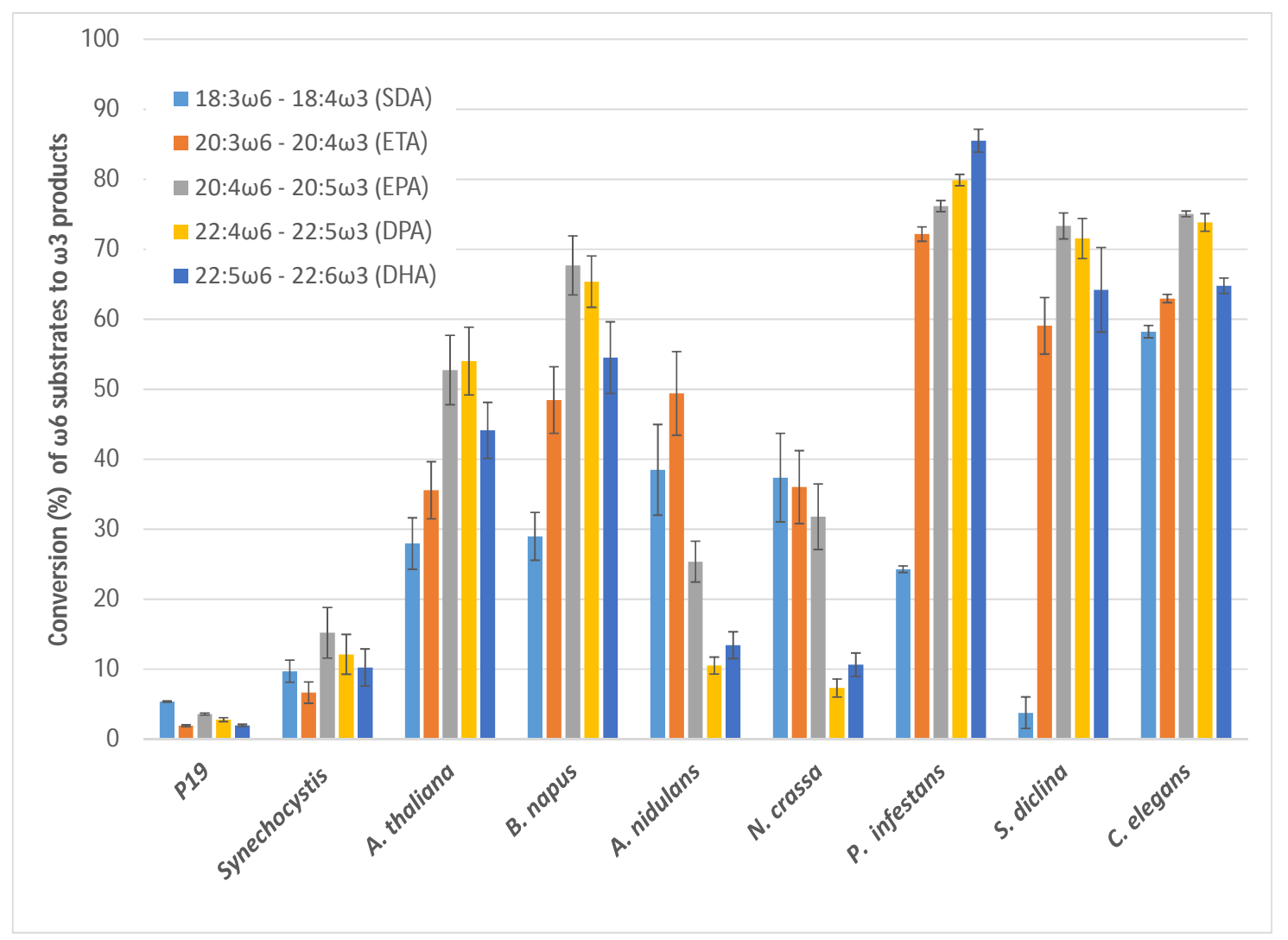

Figure 7. Competition for $\omega 6$-fatty acid substrates (18:3w6, 20:3w6, 20:4w6, 22:4w6, 22:5w6) by $\omega 3$-desaturases from various sources transiently expressed in $N$. benthamiana. Error bars represent the standard deviations of the means from triplicate assays.

\section{Discussion}

To compare the $\omega 3 \mathrm{D}$ enzymes on different $\omega 6$ fatty acid substrates, we used a $N$. benthamiana transient expression system [22] and co-infiltrated genetic constructs encoding the enzymes with the fatty acids prepared as ammonium salts. Prior to GC analysis, leaf fatty acid methyl esters (FAMEs) were purified by thin layer chromatography (TLC) to remove non-fatty acid contaminants that otherwise co-eluted with some of the FAMEs. To the best of our knowledge, this is the first report of an efficient assay of $\omega 3 \mathrm{D}$ activity in leaves. The leaf system has advantages over yeast expression systems for $\omega 3$ Ds which often fail to show activity or only reveal low activity levels in yeast cells $[16,23]$. Applying this approach, we were able to observe high and consistent levels of desaturase activity in the $N$. benthamiana leaf-based system and examine the substrate preferences of the different $\omega 3 \mathrm{Ds}$. In general, the $\omega 3 \mathrm{D}$ conversion efficiencies observed here were higher than those observed in other studies in yeast cells, and we clearly demonstrated the diversity in $\omega 3 \mathrm{D}$ activity and their broad specificity for various fatty acid substrates.

It was notable that among the eight $\omega 3 \mathrm{Ds}$ studied here, the two plant $\omega 3 \mathrm{D} s, \mathrm{At}-\omega 3 \mathrm{D}$ and Bn- $\omega 3 \mathrm{D}$, revealed the highest activities for the endogenous 18:2w6 substrate. The LC-PUFA biosynthesis

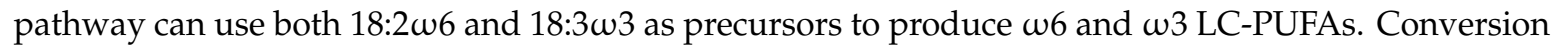
of $18: 2 \omega 6$ to $18: 3 \omega 3$ would increase the $\omega 3 / \omega 6$ ratio. The higher activity of At- $\omega 3 \mathrm{D}$ and Bn- $\omega 3 \mathrm{D}$ for 18:2w6 might imply that engineering the LC-PUFA biosynthesis pathway in these plants has the potential to enhance $\omega 3$ LC-PUFA production. In contrast, Pi- $\omega 3 \mathrm{D}$ and Sd- $\omega 3 \mathrm{D}$ from EPA-rich species did not exhibit activity on 18:2w6. Similar observations were made by others in yeast cells [11].

The specificity of the eight $\omega 3$ Ds for the $\omega 6$ fatty acid substrates tested is summarised in Supplementary Figure S1. For the 18:3w6 substrate (GLA), Ce- $\omega 3$ D had the highest activity among all the $\omega 3 \mathrm{D}$ investigated, while the plant $\omega 3 \mathrm{Ds}, \mathrm{At}-\omega 3 \mathrm{D}$ and $\mathrm{Bn}-\omega 3 \mathrm{D}$, had about half of the Ce- $\omega 3 \mathrm{D}$ 
activity. The desaturases Pi- $\omega 3 \mathrm{D}$ and Sd- $\omega 3 \mathrm{D}$ obtained from EPA-rich fungi and the animal desaturase

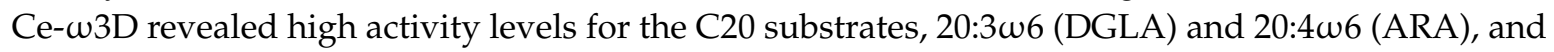
higher still for the C22 substrates, 22:4w6 (DTA) and 22:5w6 (DPAw6). Recently, Yilmaz et al. [13] also demonstrated the activities of Pi- $\omega 3 \mathrm{D}$ for C18, C20 and C22 fatty acid substrates in yeast, but in contrast to our study in leaf, observed lower activities to C22 than C20 fatty acids. On the other hand, Sd- $\omega 3 \mathrm{D}$, when expressed in yeast, exclusively desaturated C20 fatty acid substrates (5\% conversion of

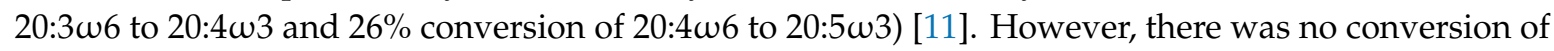
$18: 2 \omega 6$ or $18: 3 \omega 6$ to $\omega 3$ products. We also observed that the animal Ce- $\omega 3 \mathrm{D}$ had greater activities for C20 and C22 substrates than for 18:3w6, and that the level of activity was as high as for Pi- $\omega 3 \mathrm{D}$ and Sd- $\omega 3 \mathrm{D}$. This contrasted with the observations of [15] in a yeast expression system, where Ce- $\omega 3 \mathrm{~d}$ had

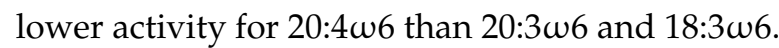

Although higher plants are devoid of C20 and C22 LC-PUFA substrates, it is interesting that At- $\omega 3 \mathrm{D}$ and Bn- $\omega 3 \mathrm{D}$ showed high activity levels with all C20 and C22 substrates tested. Also, Reed [16] demonstrated the broad specificity of Bn- $\omega 3 \mathrm{D}$ for C18-C22 fatty acids when expressed in yeast, although the activities were at low levels. In contrast, the heterologous expression in yeast of another plant $\omega 3 \mathrm{D}$ from Linum usitatissimum did not result in $\omega 3 \mathrm{D}$ activity with either 18:3w6 or

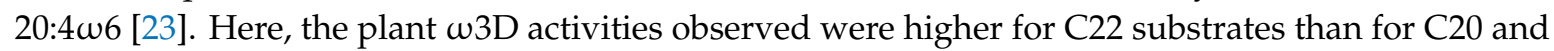
C18 substrates and were comparable to the EPA-rich fungal $\omega 3 \mathrm{Ds}$ or the animal $\omega 3 \mathrm{D}$. The activity

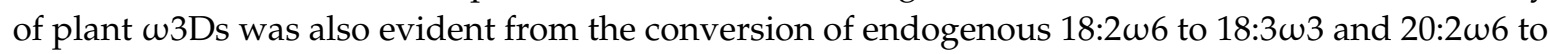
$20: 3 \omega 3$. These plant endogenous $\omega 3$ Ds might have roles in the conversion of $\omega 6$ PUFA to $\omega 3$ PUFA in DHA-producing engineered seeds, resulting in reduced levels of $\omega 6$ PUFA [6,7].

In contrast, two of the fungal desaturases, An- $\omega 3 \mathrm{D}$ and Nc- $\omega 3 \mathrm{D}$, which share close phylogeny with each other, have activities for C18 substrates that are similar to or even higher than the other w3Ds, whereas the activities were lower for $\mathrm{C} 20$ substrates and much lower for $\mathrm{C} 22$ substrates, demonstrating a clear deviation in substrate specificity for the fungal $\omega 3 \mathrm{D}$. The cyanobacterial Ss- $\omega 3 \mathrm{D}$ was unable

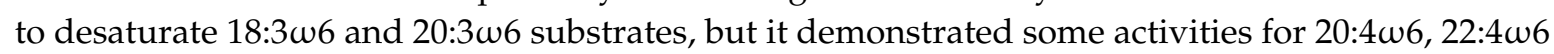
and 22:5w6.

The substrate competition assay where multiple $\omega 6$ fatty acids were supplied to these $\omega 3$ Ds

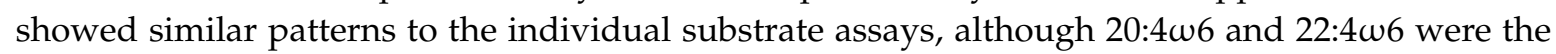
preferred substrates in the mixture for a majority of the tested enzymes, while 22:4w6 was preferred in the single substrate assays. When engineering the LC-PUFA biosynthesis pathway into crops, there would be substrate competition from different $\omega 6$ intermediates; thus, the preference of $\omega 3$ Ds for these substrates would reflect the in vivo activities better than single substrate assays. One interesting observation was that the plant $\omega 3 \mathrm{D} s, \mathrm{At}-\omega 3 \mathrm{D}$ and Bn- $\omega 3 \mathrm{D}$, had similar activity levels, and their specificities were comparable to the EPA-rich fungal Pi- and Sd- $\omega 3$ Ds and the animal Ce- $\omega 3 \mathrm{D}$, all of which exhibited higher activity for C20 and C22 LC-PUFA substrates than for C18 substrates. These have the potential to increase $\omega 3 / \omega 6$ ratios when seeking to engineer LC-PUFAs into plants for health benefits.

\section{Materials and Methods}

\subsection{Materials and Chemicals}

Fatty acid substrates were purchased from NuCheck Inc. (Elysian, MN, USA) and ammonium salts of the fatty acids were synthesised in our laboratory. Briefly, $5 \mathrm{mg}$ of fatty acid was dispersed in $0.5 \mathrm{~mL}$ of $2 \mathrm{M}$ ammonia in a $2 \mathrm{~mL}$ glass vial using a probe sonicator (Branson, Switzerland) three times for $3 \mathrm{~s}$ each, vortexing between sonications. The mixture was then incubated at $60^{\circ} \mathrm{C}$ for $20 \mathrm{~min}$. The ammonia solution was evaporated under a flow of nitrogen on a $60^{\circ} \mathrm{C}$ hot plate, and $1 \mathrm{~mL}$ of potassium-phosphate buffer was added to the vial. The salt was solubilised in the buffer by sonication. The concentration of fatty acid salt was estimated by injecting its fatty acid methyl ester into a gas chromatograph. For the preparation of the fatty acid methyl ester (FAME) of the fatty acid salt, $5 \mu \mathrm{L}$ of 
the salt solution and $5 \mu \mathrm{g}$ of heptadecanoic acid (C17:0 as internal standard) in toluene were mixed in $100 \mu \mathrm{L}$ of methanol in a $2 \mathrm{~mL}$ glass vial, and the methanol/water mixture was evaporated under a gentle flow of nitrogen, placing the vial on a $40^{\circ} \mathrm{C}$ hot plate. The fatty acid salt was incubated in $0.3 \mathrm{~mL}$ $1 \mathrm{~N}$ methanolic- $\mathrm{HCl}$ (Supelco, Castle Hill, New South Wales, Australia) at $80^{\circ} \mathrm{C}$ for $2 \mathrm{~h}$. After cooling the solution to room temperature, $0.3 \mathrm{~mL}$ of $0.9 \% \mathrm{NaCl}$ and $0.3 \mathrm{~mL}$ of hexane were added and then mixed for $5 \mathrm{~min}$. The mixture was centrifuged for $5 \mathrm{~min}$ at $1700 \times \mathrm{g}$, the upper phase of the FAME was transferred to a glass insert and the solvent was evaporated under a flow of nitrogen. The FAME was resuspended in $30 \mu \mathrm{L}$ of hexane and analysed by GC as described [24].

\subsection{Gene Constructs}

The $\omega 3$ D sequences were obtained from Synechocystis sp. [25], PMB 26:249-263), A. thaliana (Accession\# P48623, [26]), B. napus (Accession\# L01418, [27]), A. nidulans (Sequence 5 from US Patent Application No. 20060156435), N. crassa (Sequence 3 from US Patent Application No. 20060156435), P. infestans (SEQ ID NO:2 from US Patent No. 7,777,098), S. diclina (SEQ ID NO:26 from US Patent No. 7,211,656) and C. elegans (Accession\# L41807; [28]), and are designated as Ss- $\omega 3 \mathrm{D}$, At- $\omega 3 \mathrm{D}, \mathrm{Bn}-\omega 3 \mathrm{D}$, An- $\omega 3 \mathrm{D}, \mathrm{Nc}-\omega 3 \mathrm{D}, \mathrm{Pi}-\omega 3 \mathrm{D}, \mathrm{Sd}-\omega 3 \mathrm{D}$ and Ce- $\omega 3 \mathrm{D}$, respectively. The coding sequences were synthesised at GeneArt (Thermo Fisher Scientific) with codon optimisation and cloned into the binary vector pJP3343 [29] under control of the CaMV 35S promoter.

\subsection{Transient Expression in N. benthamiana Leaf and Fatty Acid Analysis}

Agrobacterium tumefaciens strain AGL1 was separately transformed with the gene constructs. Transformed cells were co-infiltrated with AGL1 containing a viral silencing protein (p19) gene into $N$. benthamiana leaves as described before [22] with some modifications. In brief, AGL1 cultures were grown overnight at $28^{\circ} \mathrm{C}$ in LB broth containing appropriate antibiotics. Acetosyringone $(0.1 \mathrm{mM})$ was added to the cultures, which were grown for a further $3 \mathrm{~h}$ before the cells were pelleted down and resuspended in infiltration buffer ( $5 \mathrm{mM} 4$-morpholineethanesulfonic acid, $5 \mathrm{mM} \mathrm{MgSO}_{4}$ ).

The solubilized fatty acid salts were added in the infiltration buffer at the final concentration of $1 \mathrm{mM}$ each for single substrate assay or $0.5 \mathrm{mM}$ each for competition assay with each Agrobacterium culture at $\mathrm{OD}_{600}$ of 0.1 . Approximately $0.1 \mathrm{~mL}$ of the appropriate mixture was infiltrated in each spot with a needleless syringe on the undersides of leaves of five-week-old plants. After 3 days in a growth chamber, the infiltrated leaf tissues were collected into $2 \mathrm{~mL}$ glass vials and washed once with $0.2 \%$ tween and twice with water to wash off any remaining fatty acid substrate(s) from the leaf surface. The leaf samples were dried in a freeze dryer overnight, and fatty acid methyl esters were prepared as mentioned above. To remove non-fatty acid leaf contaminants from the FAMEs, FAME samples were applied to a TLC plate (Silica gel-60, MERCK, Castle Hill, New South Wales, Australia) and run in a mixture of hexane/diethyl ether/acetic acid $(80 / 20 / 1, v / v / v)$. FAME bands were viewed under UV after spraying the plates with $0.01 \%$ primuline in acetone/water $(8 / 2, v / v)$. The bands were collected into GC vials and FAMEs were extracted from the silica using hexane/ diethyl ether $(1 / 3, v / v)$ and analysed by GC as described [24].

\section{Conclusions}

In conclusion, $\omega 3 \mathrm{Ds}$ show activity on a range of C18-C22 $\omega 6$ fatty acids and a diversity of substrate preference and level of activity in producing $\omega 3$ LC-PUFAs. This will be important for EPA and DHA production in heterologous hosts. Of particular interest, the endogenous plant w3Ds had previously unsuspected activities on a wide range of $\omega 6$ fatty acid substrates, especially on the LC-PUFA substrates. This would enhance the ability to engineer oil crops as alternative sources of $\omega 3$ LC-PUFAs.

Supplementary Materials: Supplementary materials can be found at http://www.mdpi.com/1422-0067/20/12/ 3058/s1. 
Author Contributions: Conception: J.P., R.d.F., and S.S.; experiment design: P.S., S.S., X.-R.Z., and R.d.F.; methodology: P.S. and X.-R.Z.; experimentation: P.S. and S.V.P.; data analysis: all authors; writing: P.S., X.-R.Z., R.d.F., and S.S., with input from all authors.

Funding: This research was funded by CSIRO Grant.

Acknowledgments: We thank Cheryl Blundell for excellent technical support.

Conflicts of Interest: The authors declare no conflict of interest.

\section{Abbreviations}

\begin{tabular}{|c|c|}
\hline ALA & $\alpha$-linolenic acid (18:3w3) \\
\hline An & Aspergillus nidulans \\
\hline ARA & 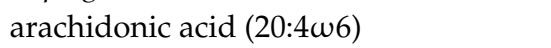 \\
\hline At & Arabidopsis thatiana \\
\hline $\mathrm{Bn}$ & Brassica napus \\
\hline $\mathrm{Ce}$ & Caenorhabditis elegans \\
\hline DGLA & dihomo gamma-linolenic acid (20:3w6) \\
\hline DHA & docosahexaenoic acid (22:6w3) \\
\hline DPA & docosapentaenoic acid (22:5w3) \\
\hline DPA-6 & docosapentaenoic acid (22:5w6) \\
\hline DTA & docosatetraenoic acid $(22: 4 \omega 6)$ \\
\hline EPA & eicosapentaenoic acid (20:5w3) \\
\hline ETA & eicosatetraenoic acid (20:4w3) \\
\hline FAME & fatty acid methyl ester \\
\hline GC & gas chromatography \\
\hline GLA & gamma-linolenic acid (18:3w6) \\
\hline LA & linoleic acid (18:2w6) \\
\hline LC-PUFA & long chain-polyunsaturated fatty acid \\
\hline $\mathrm{Nc}$ & Neurospora crassa \\
\hline$\omega 3 \mathrm{D}$ & omega-3 desaturase \\
\hline $\mathrm{Pi}$ & Phytophthora infestans \\
\hline SDA & stearidonic acid \\
\hline Ss & Synechocystis sp. \\
\hline Sd & Saprolegnia diclina \\
\hline TLC & thin layer chromatography \\
\hline
\end{tabular}

\section{References}

1. Simopoulos, A.P. An Increase in the Omega-6/Omega-3 Fatty Acid Ratio Increases the Risk for Obesity. Nutrients 2016, 8, 128. [CrossRef] [PubMed]

2. $\quad$ Berger, M.E.; Smesny, S.; Kim, S.W.; Davey, C.G.; Rice, S.; Sarnyai, Z.; Schlöegelhofer, M.; Schafer, M.R.; Berk, M.; McGorry, P.D.; et al. Omega-6 to omega-3 polyunsaturated fatty acid ratio and subsequent mood disorders in young people with at-risk mental states: A 7-year longitudinal study. Transl. Psychiatry 2017, 7, e1220. [CrossRef] [PubMed]

3. Ruxton, C.H.; Reed, S.C.; Simpson, M.J.; Millington, K.J. The health benefits of omega-3 polyunsaturated fatty acids: A review of the evidence. J. Hum. Nutr. Diet. 2004, 17, 449-459. [CrossRef] [PubMed]

4. Zhang, Z.; Fulgoni III, V.L.; Kris-Etherton, P.M.; Mitmesser, S.H. Dietary Intakes of EPA and DHA Omega-3 Fatty Acids among US Childbearing-Age and Pregnant Women: An Analysis of NHANES 2001-2014. Nutrients 2018, 10, 416. [CrossRef] [PubMed]

5. Usher, S.; Han, L.; Haslam, R.P.; Michaelson, L.V.; Sturtevant, D.; Aziz, M.; Chapman, K.D.; Sayanova, O.; Napier, J.A. Tailoring seed oil composition in the real world: Optimising omega-3 long chain polyunsaturated fatty acid accumulation in transgenic Camelina sativa. Sci. Rep. 2017, 7, 6570. [CrossRef] [PubMed]

6. Petrie, J.R.; Shrestha, P.; Belide, S.; Kennedy, Y.; Lester, G.; Liu, Q.; Divi, U.K.; Mulder, R.J.; Mansour, M.P.; Nichols, P.D.; et al. Metabolic engineering Camelina sativa with fish oil-like levels of DHA. PLoS ONE 2014, 9, e85061. [CrossRef] [PubMed] 
7. Petrie, J.R.; Shrestha, P.; Zhou, X.-R.; Mansour, M.P.; Liu, Q.; Belide, S.; Nichols, P.D.; Singh, S.P. Metabolic engineering plant seeds with fish oil-like levels of DHA. PLoS ONE 2012, 7, e49165. [CrossRef] [PubMed]

8. Wang, M.; Chen, H.; Gu, Z.; Zhang, H.; Chen, W.; Chen, Y.Q. Omega3 fatty acid desaturases from microorganisms: Structure; function; evolution; and biotechnological use. Appl. Microbiol. Biotechnol. 2013, 97, 10255-10262. [CrossRef]

9. Damude, H.G.; Zhang, H.; Farrall, L.; Ripp, K.G.; Tomb, J.F.; Hollerbach, D.; Yadav, N.S. Identification of bifunctional $\Delta 12 / \omega 3$ fatty acid desaturases for improving the ratio of $\omega 3$ to $\omega 6$ fatty acids in microbes and plants. Proc. Natl. Acad. Sci. USA 2006, 103, 9446-9451. [CrossRef]

10. Sakuradani, E.; Abe, T.; Iguchi, K.; Shimizu, S. A novel fungal w3-desaturase with wide substrate specificity from arachidonic acid-producing Mortierella alpina 1S-4. Appl. Microbiol. Biotechnol. 2005, 66, 648-654. [CrossRef]

11. Pereira, S.L.; Leonard, A.E.; Huang, Y.S.; Chuang, L.T.; Mukerji, P. Identification of two novel microalgal enzymes involved in the conversion of the omega 3-fatty acid; eicosapentaenoic acid; into docosahexaenoic acid. Biochem. J. 2004, 384, 357-366. [CrossRef] [PubMed]

12. Bostock, R.M.; Kuc, J.A.; Laine, R.A. Eicosapentaenoic and arachidonic acids from Phytophthora infestans elicit fungitoxic sesquiterpenes in the potato. Science 1981, 212, 67-69. [CrossRef] [PubMed]

13. Yilmaz, J.L.; Lim, Z.L.; Beganovic, M.; Breazeale, S.; Andre, C.; Stymne, S.; Vrinten, P.; Senger, T. Determination of substrate preferences for desaturases and elongases for production of docosahexaenoic acid from oleic acid in engineered canola. Lipids 2017, 52, 207-222. [CrossRef] [PubMed]

14. Wallis, J.G.; Watts, J.L.; Browse, J. Polyunsaturated fatty acid synthesis: What will they think of next? Trends Biochem. Sci. 2002, 27, 467. [CrossRef]

15. Meesapyodsuk, D.; Reed, D.W.; Savile, C.K.; Buist, P.H.; Ambrose, S.J.; Covello, P.S. Characterization of the regiochemistry and cryptoregiochemistry of a Caenorhabditis elegans fatty acid desaturase (FAT-1) expressed in Saccharomyces cerevisiae. Biochemistry 2000, 39, 11948-11954. [CrossRef] [PubMed]

16. Reed, D.W.; Schafer, U.A.; Covello, P.S. Characterization of the Brassica napus extraplastidial linoleate desaturase by expression in Saccharomyces cerevisiae. Plant Physiol. 2000, 122, 715-720. [CrossRef] [PubMed]

17. Hoffmann, M.; Hornung, E.; Busch, S.; Kassner, N.; Ternes, P.; Braus, G.H.; Feussner, I. A small membrane-peripheral region close to the active center determines regioselectivity of membrane-bound fatty acid desaturases from Aspergillus nidulans. J. Biol. Chem. 2007, 282, 26666-26674. [CrossRef] [PubMed]

18. Goodrich-Tanrikulu, M.; Stafford, A.E.; Jacobson, D.J. A systematic survey of the fatty acid composition of Neurospora strains. Fungal Genet. Rep. 1999, 46, 3. [CrossRef]

19. Chen, G.; Qu, S.; Wang, Q.; Bian, F.; Peng, Z.; Zhang, Y.; Ge, H.; Yu, J.; Xuan, N.; Bi, Y.; et al. Transgenic expression of delta-6 and delta-15 fatty acid desaturases enhances omega-3 polyunsaturated fatty acid accumulation in Synechocystis sp. PCC6803. Biotechnol. Biofuels 2014, 7, 1-10. [CrossRef]

20. Kikukawa, H.; Sakuradani, E.; Kishino, S.; Park, S.-B.; Ando, A.; Shima, J.; Ochiai, M.; Shimizu, S.; Ogawa, J. Characterization of a trifunctional fatty acid desaturase from oleaginous filamentous fungus Mortierella alpina 1S-4 using a yeast expression system. J. Biosci. Bioeng. 2013, 116, 672-676. [CrossRef]

21. Chang, K.J.L.; Mansour, M.P.; Dunstan, G.A.; Blackburn, S.I.; Koutoulis, A.; Nichols, P.D. Odd-chain polyunsaturated fatty acids in thraustochytrids. Phytochemistry 2011, 72, 1460-1465. [CrossRef]

22. Wood, C.C.; Petrie, J.R.; Shrestha, P.; Mansour, M.P.; Nichols, P.D.; Green, A.G.; Singh, S.P. A leaf-based assay using interchangeable design principles to rapidly assemble multistep recombinant pathways. Plant Biotechnol. J. 2009, 7, 914-924. [CrossRef] [PubMed]

23. Vrinten, P.; Hu, Z.; Munchinsky, M.A.; Rowland, G.; Qiu, X. Two FAD3 desaturase genes control the level of linolenic acid in flax seed. Plant Physiol. 2005, 139, 79-87. [CrossRef] [PubMed]

24. Zhou, X.-R.; Green, A.G.; Singh, S.P. Caenorhabditis elegans $\Delta 12$-desaturase FAT-2 is a bifunctional desaturase able to desaturate a diverse range of fatty acid substrates at the $\Delta 12$ and $\Delta 15$ positions. J. Biol. Chem. 2011, 286, 43644-43650. [CrossRef] [PubMed]

25. Sakamoto, T.; Wada, H.; Nishida, I.; Ohmori, M.; Murata, N. Delta 9 acyl-lipid desaturases of cyanobacteria. Molecular cloning and substrate specificities in terms of fatty acids; sn-positions; and polar head groups. J. Biol. Chem. 1994, 269, 25576-80157. [PubMed]

26. Yadav, N.S.; Wierzbicki, A.; Aegerter, M.; Caster, C.S.; Pérez-Grau, L.; Kinney, A.J.; Hitz, W.D.; Booth Jr, J.R.; Schweiger, B.; Stecca, K.L.; et al. Cloning of higher plant $\omega$-3 fatty acid desaturases. Plant Physiol. 1993, 103, 467-476. [CrossRef] [PubMed] 
27. Arondel, V.; Lemieux, B.; Hwang, I.; Gibson, S.; Goodman, H.M.; Somerville, C.R. Map-based cloning of a gene controlling omega-3 fatty acid desaturation in Arabidopsis. Science 1992, 258, 1353-1355. [CrossRef]

28. Spychalla, J.P.; Kinney, A.J.; Browse, J. Identification of an animal omega-3 fatty acid desaturase by heterologous expression in Arabidopsis. Proc. Natl. Acad. Sci. USA 1997, 94, 1142-1147. [CrossRef]

29. Reynolds, K.; Taylor, M.; Zhou, X.-R.; Vanhercke, T.; Wood, C.; Blanchard, C.; Singh, S.; Petrie, J.R. Metabolic engineering of medium-chain fatty acid biosynthesis in Nicotiana benthamiana plant leaf lipids. Front. Plant Sci. 2015, 6, 164. [CrossRef]

(C) 2019 by the authors. Licensee MDPI, Basel, Switzerland. This article is an open access article distributed under the terms and conditions of the Creative Commons Attribution (CC BY) license (http://creativecommons.org/licenses/by/4.0/). 